\title{
RFID TAGS USED IN PREVENTIVE MAINTENANCE PROGRAMME FOR MOTORS AND GENERATORS
}

\author{
Tracy Aleong1,* and Kit Fai Pun ${ }^{2}$ \\ ${ }^{1,2}$ Department of Mechanical \& Manufacturing Engineering, Faculty of Engineering, \\ The University of the West Indies, Trinidad and Tobago, West Indies \\ ${ }^{1}$ Email: tracy.aleong1@gmail.com; tracy.aleong@my.uwi.edu \\ ${ }^{2}$ Email: KitFai.Pun@sta.uwi.edu
}

\begin{abstract}
In modern plant operations, a well-planned preventive maintenance programme is vital to reliable and long-life operation of motors and generators. Many companies lack such a programme due to the high cost and intricacy associated with employing major sensing techniques for monitoring live equipment status. This paper presents the findings of a pilot project that was to design a low cost Radio Frequency Identification (RFID) based system paired with the Internet of Things (IoT) communications to create a preventive maintenance programme that could provide real-time updates for installed motors and generators at a particular site. The basic system would incorporate ultra-high frequency RFID sensor tags for monitoring vibration and temperature, fixed RFID readers with antennas, and a based unit (built around a personal computer) with access to the internet. This is an on-line RFID-based monitoring system. The RFID tags would allow the connection of the physical assets to the digital world having a direct effect to a company's business drivers such as cost savings, greater operational efficiency, and increased productivity. Installing the basic system on a trial basis is recommended. It is anticipated that, the RFID based system could provide a long-term solution to the current problems of complex and expensive data collection
\end{abstract}

Keywords: RFID, Internet of Things, sensors, maintenance, productivity.

https://doi.org/10.47412/NCPE8453

\section{Introduction}

Radio Frequency Identification (RFID) is a form of wireless communication that incorporates the use of electromagnetic or electrostatic coupling in the radio frequency portion of the electromagnetic spectrum to uniquely identify an object, animal or person [1]. Traditionally, RFID tags were used in applications such as tracking of inventory, assets, personnel, and manufacturing of goods and materials. In recent years, this system has been combined with the use of smart sensor technology, which allows the possibility of measuring system parameters such as resistance, temperature, vibration, pressure and movement [2]. This measured data can then be transmitted wirelessly through such mediums as the internet of things (IoT) for remote viewing and analysing [3].

This paper will look at integrating RFID sensor tag to create a condition-based/preventative maintenance based monitoring programme for electric rotating machine such as motors and generators. It covers the basic system components required to have an operational system capable of collecting temperature and vibration readings on both rotary and stationary parts of the machine. The information obtainable from condition monitoring can be used to develop a preventative maintenance programme for those operating field units. The benefits of such programmes sufficiently justifies the case to implement a system capable 
of providing the sensor data used to analyse how the motors and generators in current operations are performing at any given time.

In this paper, a brief literature review on RFID is covered in section 2, followed by section 3 providing an understanding on the topic of condition monitoring using process measurement of temperature and vibrations. Section 4 dives into the RFID system components and requirements needed to implement such a system and ends with a short conclusion in section 5 .

\section{Literature review}

For the perspective of field application, the current categorisation of RFID sensors and system variables to be measured are best presented by Meng and Li [4]. Figure 1 provides an adequate depiction of the everyday opportunities that can use this technology.

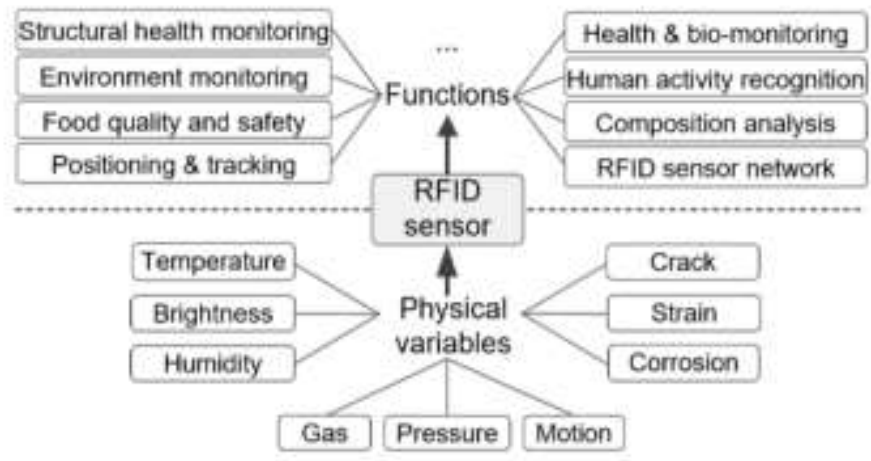

Figure 1: Applications of RFID sensors [4]

Condition-based maintenance is a novel scientific maintenance strategy, which decides the optimal maintenance time according to the actual condition of the machinery to prevent unexpected catastrophic faults, improve the reliability and availability of the machinery, decrease downtime, and increase operating efficiency [5,6]. Techniques used in condition monitoring has evolved overtime to make the best use of the latest technological advances, from the earliest watching, listening, and feeling of skilled engineers, analog instruments, portable data loggers, and analysers to online-wired and wireless monitored systems [5]. Employing such a programme allows industry operators to develop predictive maintenance actions for many installed electrical rotating machines. In some motors and generators, vibration and temperature parameters are monitored by wired sensors and the information collected is used to provide warning signals or shut down the system before any catastrophic failure occurs[7].

\section{Condition Monitoring of Motors and Generators}

High temperatures and excessive vibrations are known to be some of the reasons why electrical rotating equipment expectantly shutdown, regardless of specific type. The dynamic balance of the rotating components significantly affects the operational life span of rotating equipment. When the rotating mass becomes unbalanced, the level of vibration and potentially the thermal signature changes in relation to the level of imbalance within the rotating equipment. On the other hand, overheating is relatively common for a number of reasons, i.e. overload, poor power condition, high effective service factor, frequent stops and starts or environmental reasons.

There are different parts of a rotating machine that sensors can be placed to monitor for temperature and vibrations as seen in Fig. 1. However, mounting traditional wired sensors are limited to the stationary parts of the unit whilst the rotary or moving parts left unmonitored. Wireless sensing solutions fill the gap for 
these areas but usually carries a high operational and installation cost as the main source of power is derived from a battery, that would require routine change outs.

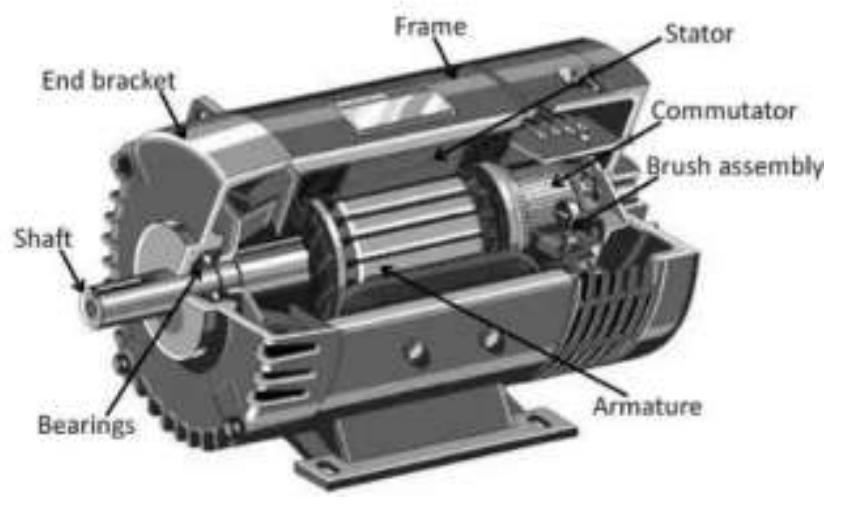

Figure 2: A Cutaway view of a typical electric motor [8]

\subsection{Condition Based Monitoring - Temperature Measurements}

Temperature detection has repeatedly been shown to be an effective global monitoring technique for most electrical machines, using simple sensors that can aid in preventing the occurrence of overheating. In both induction and synchronous electric motors and generators, excessive heat causes rapid deterioration of insulation windings. The electrical insulation in rotating machine stator and rotor windings of a synchronous unit is essential to the continued functioning. The degree rule applies to motors operating at above rated temperature; insulation life drops by half for each $10^{\circ} \mathrm{C}$ of over temperature [9].

A combination of thermal, electrical and mechanical stresses coupled with environmental stresses causes the windings to age over time. The failure of the insulation can take years under these aging stresses before it occurs. This long time between the initiation of a failure process, and when the failure actually occurs, makes testing and monitoring of machine insulation particularly effective for anticipating the unexpected breakdowns of individual units. Apart from the expense incur for repairing the damage equipment, companies also experience economic loss due to the interruption of the plant operation. For this reason, management recognises the advantages of implementing preventative maintenance programmes for critical plant equipment.

In the case of machines using permanent magnets, high temperature can cause loss of magnetization so most motors and generators are designed with the magnets oversized to prevent this from happening. Magnets that have been demagnetised cannot regain its original magnetic strength even after conditions have normalised. Therefore, monitoring the temperature in these units are critical to preserving the continued operation in the field.

\subsection{Condition Based Monitoring - Mechanical Vibration}

The vibration of any object in motion is characterised by variations of amplitude, intensity, and frequency. Vibration analysis is a process of looking for anomalies and monitoring change from the established vibration signature of a system that can be later correlated to a physical phenomenon and then used to assess the health of the equipment [10]. Vibration monitoring usually involves a non-invasive sensing method, which utilises a number of specialised sensors and broad bandwidth with complex analysis. Sensors are usually placed in regions of the machine where it can measure vibration of the bearings, gearbox and rotor parts of the unit. Online monitoring of these areas in motors and generators can help identify various undesirable conditions including unbalance, misalignment, looseness, bearing wear, rubbing, hydraulic 
problems, electrical problems, resonance, and eccentricity of rotating parts. If faults are detected early, preventative maintenance can be employed to reduce further damage of the unit.

\subsubsection{Bearing Condition Monitoring}

Vibration within the machine is often the source of bearing faults. Monitoring bearing conditions can provide the maintenance team with the data and justification required for conducting repairs or replacements. Other Bearing defects can arise from excessive loads, true or false brinelling, overheating, reverse loading, normal fatigue failure, corrosion, loose or tight fits, and misalignment, amongst other.

\subsubsection{Gearbox Vibration Monitoring}

Measuring the vibration within the gearbox is often performed in condition monitoring programmes. In gearboxes, constant impacting and friction can occur and with just a single crack occurring in a gear, can cause a slight change in speed once the defective teeth are inside of the load zone. This will result in impacting, and if there is insufficient lubrication for the gear teeth, friction will also occur.

\subsubsection{Rotor Vibration Monitoring}

Lateral vibrations in rotors can arise because of many faults such as instability and unbalance, impacting forces on the rotor. This can result in cracks, which directly affects the natural frequencies because of reduced rigidness. Proper rotor vibration analysis can monitor the rotor's behaviour to help locate a developed crack.

\section{Condition Monitoring Data Acquisition using a RFID Sensor Tag System}

Many manufacturers of rotating equipment uses battery operated wireless sensors for measuring temperature and vibration directly on the rotor and shaft during the testing phase to ensure product performance and adherence to specifications. This usually occurs before the unit is shipped out of the factory, therefore installing those types of sensors are quick and easy to complete in comparison to wired sensors over the brushing, which can be very complex. The battery life does not pose an issue to the operation, as the testing is short lived. However, in the case of continuous operation testing for the purpose of condition monitoring, wireless sensors are not the optimal choice. This is due to the frequent battery changes resulting in process interruptions to allow change out and higher operational cost.

\subsection{System Components}

The components required to make up the system design for using RFID sensor tags for monitoring the temperature and vibrations within a electric motor or generator are as follows:

- RFID Sensor Tag or Transponder- for measuring temperature or vibration on metallic surfaces

- RFID Reader -Compatible handheld (mobile) Reader with integral antenna

- Logging Station -Compatible PC/Laptop/Tablet \& proprietary software (depending on Supplier of Tag and reader) and IoT communication

\subsubsection{RFID Sensor Tag or Transponder}

An RFID tag in its most simplistic form comprises of three parts; an antenna for transmitting and receiving signals; an RFID chip (or integrated circuit, IC) which stores the tag's ID and other information; and lastly, some form of encapsulation [11, 12]. Passive tags refers to those tags that have no internal power source such as a battery. They are widely used in comparison to those that have an internal battery primarily because they are smaller, thinner, longer lasting and less expensive to implement. These tags rely on an external source to be "powered up". This is achieved by the electromagnetic field (EMF) created by the RFID reader provided it is within the reading range. When the tag receives the transmission from the reader/antenna, the tag's antenna captures coupled energy from the EMF and transfers the tag's ID (the 
tag's IC coordinates this process). The coupled energy is rectified and the voltage multiplied via a multistage Greinacher half-wave rectifier to power up the IC. The energised chip modulates the energy with the desired information and then transmits a signal back towards the antenna of the reader [13].The sensor tag versions operate in the same manner, as RF energy is harvested from the RF radiation emitted by the reader to power the circuit and complete the sensing. The inductive coupling or backscattered radio wave in RF identification is used to detect the physical parameters of tagged objects, hence the ability to work as a wireless sensor. A compact design for a RFID temperature sensor tag and vibration sensor tag is seen in Fig. 3 a \& b.

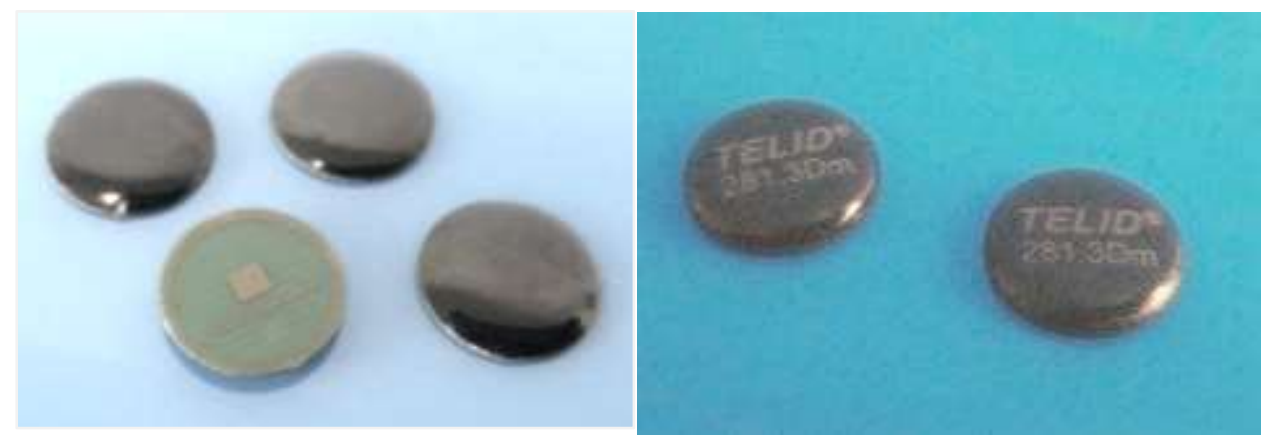

Figure 3: a) RFID temperature sensor tag, b) RFID vibration sensor tag [14]

As with traditional wired sensors, selecting the appropriate measurement range required for temperature and vibration sensor tags must be accurate. The information for both temperature and vibration operating limits inclusive of maximum and minimum values can be acquired from the manufacturer of the respective electric rotating unit or the product data sheet if available. The temperature operating range is usually given in degrees Celsius (deg C) or degrees Fahrenheit (deg F) whilst the vibration values are Frequency measured in Hertz $(\mathrm{Hz})$ and Acceleration from gravity $(\mathrm{g})$. Knowing these values will allow the selection for an appropriate sensor tag capable of measuring a fault condition.

\subsubsection{RFID Reader}

The main functions of an RFID reader (Fig. 4) is to collect the data stored in the tag and provide power through the emission of radio waves. The reader emits radio waves in ranges of anywhere from one (1) inch to one hundred (100) feet or more, depending upon its power output and the radio frequency user. When an RFID tag passes through the electromagnetic zone or read range, it detects the reader's activation signal. The reader decodes the data encoded in the tag's IC and the data is passed to the host computer for storage or processing.

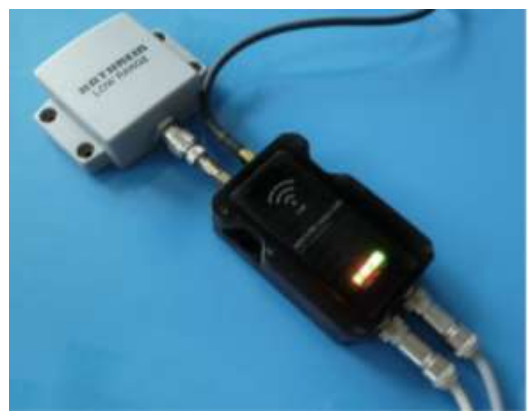

Figure 4: RFID Reader [14]

Readers can be installed in closed proximity to the where the tags are mounted, this would provide optimum communication between tags and reader, or further away but within the read distance limit as specified by the manufacturer of the device. 


\subsubsection{Logging Station}

To complete the system, information collected by the reader is transmitted to a user-friendly host for storing and future analysing. In most cases, this is a compatible PC/Laptop/Tablet capable of installing and running proprietary software provided by the supplier of the tags and reader. The suppliers for the RFID components usually give system requirements. The reader can be connected directly to the computer via USB or serial either for transferring information or if available, through Bluetooth communication. In the case where the reader is mounted close to the installed sensor tags, using a more portable host such as a tablet is easier to operate. The availability of internet connection opens the opportunity for remote access and sharing of information to key stakeholders and consultation with data analysing specialist on.

\subsection{System Arrangement}

Whilst selecting the basic system components, consideration should be given on potential mounting locations for the sensor tags. Having proper measurement coverage is important to achieving effective condition analysis. Figure 5 shows the distribution of temperature sensor tags on the rotor of motor. Mounting sensors on the frame and other parts accessible can be considered. The more sensor tags used, the more information is collected that can build a thermal map of the rotor and other parts of the unit. For vibration monitoring, section 3.2 identified three main areas of importance to monitor; bearing gearbox and rotor for rotating machines.

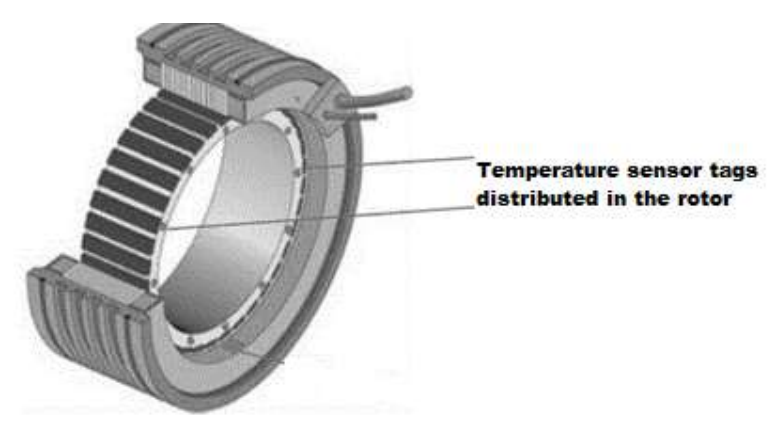

Figure 5: RFID Sensor tags installed on rotor [15]

\subsection{Testing and Operations}

With the system components installed, all that is left should be to start up the unit and allow the system to collect initial data/readings to create a baseline signature for each unit. This baseline will be used for referencing and developing a predictive/preventative maintenance programme.

\subsection{Limitations of Using System in an Industrial Environment}

As with most technological systems, there are limiting factors for use of RFID sensor tags in field application. The following applies to an industrial environment setting and must be considered in determining suitability:

- Design and installation - Passive tags will not operate unless it is within the range of the reader so that it can scavenge power and perform its function. Read distance must be considered when determining the optimal position for mounting the reader so that it is within the transmission range of the field transponders. If reader has to be placed in the field, then proper approval and certification must be adhered too depending on the company's requirements.

- Frequency - Choosing the optimal frequency depends on various factors, such as transmission mode and behavior of tagged goods, read/receive distances and the environment. Properties of some materials 
such as goods containing water or metal surface may be an obstacle to RFID application with the risk of signal absorption or reflections respectively at a given frequency, which can lead to corrupted data transmission.

- Standardization of Frequencies - There are different versions of RFID sensor tags and readers currently available, operating at different frequencies. Using a combination of sensor tags and reader from different suppliers may encounter interoperability issues resulting in conflicts with communication protocols, signal modulation types, data transmission rates and data encoding.

- Environmental Effects - Systems that work perfectly in a lab setting may encounter problems when faced with environmental noise, interference, or human elements [16]. For example, simply repositioning or re-aligning readers would often address performance issues.

- Security and privacy issues - are major areas of concern expressed for determining the suitability of this technology in different applications. The problem lies in that radio-based technology mode of communication via invisible wireless channels are undetectable. Therefore, some tag designs would promiscuously surrender their identity when queried by any reader operating at the appropriate frequency.

- Social and legal issues - certain countries have restrictions on the allocation of the electromagnetic spectrum and may prohibit the use of these systems. In addition, there may be concerns about the health implications from continuous exposure to electro-magnetic waves that prohibit the progress of getting these systems accepted for use.

- Data Collision - Attempting to read several tags at a time may result in a signal collision and ultimately to data loss. To prevent this, anti-collision algorithms (most of them are patented or patent-pending) can be applied at an extra cost.

\section{Conclusions}

The basic RFID Sensor tag and system components that can be used for condition based monitoring of electrical rotating equipment are presented in this paper. The sensor tags discussed are those used for measuring temperature and vibrations respectively. Overheating and excessive vibrations are common problems likely to affect any motor or generator at some point during their lifetime. Performing predictive maintenance through motor temperature and vibration monitoring can prevent issues resulting from a variety of motor faults, including those often found in motor bearings, gearboxes and rotors. The importance of developing a preventative maintenance programme through condition monitoring of these two process variables are highlighted to some extent within the paper. The used of RFID sensor tag for this application was chosen as the technology is completely autonomous, energy-independent, inexpensive, and easy to install. This makes it a higher potential option for implementing it as part of a system dedicated to preventive maintenance of motors and generators especially those currently operating in the field without any form of performance analysis.

\section{References}

[1] P. Kuppusamy, "Smart Education Using Internet of Things Technology," in Emerging Technologies and Applications in Data Processing and Management: IGI Global, 2019, pp. 385412.

[2] H. G. Bilic, T. Buyukoztekin, and S. Ozdemir, "The Use of Chipless Sensors with RFID for Condition Monitoring," in 2018 International Conference on Artificial Intelligence and Data Processing (IDAP), 2018: IEEE, pp. 1-4.

[3] K. Finkenzeller, RFID handbook: fundamentals and applications in contactless smart cards, radio frequency identification and near-field communication. John Wiley \& Sons, 2010. 
[4] Z. Meng and Z. Li, "RFID Tag as a Sensor-a review on the innovative designs and applications," Measurement science review, vol. 16, no. 6, pp. 305-315, 2016.

[5] L. Hou and N. W. Bergmann, "Novel industrial wireless sensor networks for machine condition monitoring and fault diagnosis," IEEE transactions on instrumentation and measurement, vol. 61, no. 10, pp. 2787-2798, 2012.

[6] G. Stone, "Condition monitoring and diagnostics of motor and stator windings-A review," IEEE Transactions on Dielectrics and Electrical Insulation, vol. 20, no. 6, pp. 2073-2080, 2013.

[7] S. Korkua, H. Jain, W.-J. Lee, and C. Kwan, "Wireless health monitoring system for vibration detection of induction motors," in 2010 IEEE Industrial and Commercial Power Systems Technical Conference-Conference Record, 2010: IEEE, pp. 1-6.

[8] I. McKenzie. "What is the relationship between back EMF, flux, and the speed of a DC motor?" https://www.quora.com/What-is-the-relationship-between-back-EMF-flux-and-the-speed-of-aDC-motor (accessed February, 2020).

[9] P. Engineering. "When it comes to motors, how hot is hot?" https://www.plantengineering.com/articles/when-it-comes-to-motors-how-hot-is-hot/ (accessed 02/25, 2020).

[10] Dynapar. "Vibration Analysis \& Vibration Monitoring " https://www.dynapar.com/Technology/Vibration-Analysis (accessed 25- Feb, 2020).

[11] M. S. S. Hossain and N. Karmakar, "An overview on RFID frequency regulations and antennas," in 2006 International Conference on Electrical and Computer Engineering, 2006: IEEE, pp. 424427.

[12] R. Want, "An Introduction to RFID Technology, IEEE Pervasive Computing," ed: Jan, 2006.

[13] M. Kaur, M. Sandhu, N. Mohan, and P. S. Sandhu, "RFID technology principles, advantages, limitations \& its applications," International Journal of Computer and Electrical Engineering, vol. 3, no. 1, p. 151, 2011.

[14] MicroSensys. "RFID in Motion." https://www.microsensys.de/en/ (accessed 03 February, 2020).

[15] M. Choperena. "RFID-based sensors as a data source for the IoT." https://www.smart2zero.com/news/rfid-based-sensors-data-source-iot/page/0/1 (accessed 03 February, 2020).

[16] S. A. Weis, "RFID (radio frequency identification): Principles and applications," System, vol. 2, no. 3, pp. 1-23, 2007. 